\title{
Orbit Hemangiopericytoma
}

National Cancer Institute

\section{Source}

National Cancer Institute. Orbit Hemangiopericytoma. NCI Thesaurus. Code C4547.

A benign or malignant hemangiopericytoma arising from the orbit. 